https://helda.helsinki.fi

Outcomes of surgery for necrotizing enterocolitis and spontaneous intestinal perforation in Finland during 1986-2014

Karila, Kristiina

2018-10

Karila , K , Anttila , A , Iber , T , Pakarinen , M \& Koivusalo , A 2018 , ' Outcomes of surgery for necrotizing enterocolitis and spontaneous intestinal perforation in Finland during 1986-2014 ' , Journal of Pediatric Surgery , vol. 53 , no. 10 , pp. 1928-1932 . https://doi.org/10.1016/j.jpedsurg.2018.

http://hdl.handle.net/10138/305689

https://doi.org/10.1016/j.jpedsurg.2018.07.020

publishedVersion

Downloaded from Helda, University of Helsinki institutional repository.

This is an electronic reprint of the original article.

This reprint may differ from the original in pagination and typographic detail.

Please cite the original version. 


\title{
Outcomes of surgery for necrotizing enterocolitis and spontaneous intestinal perforation in Finland during 1986-2014
}

\author{
Kristiina Karila ${ }^{\mathrm{a}, *}$, Annaleena Anttila ${ }^{\mathrm{b}}$, Tarja Iber ${ }^{\mathrm{b}}$, Mikko Pakarinen ${ }^{\mathrm{a}}$, Antti Koivusalo ${ }^{\mathrm{a}}$ \\ a Children's Hospital, University of Helsinki, Finland \\ ${ }^{\mathrm{b}}$ Children's Hospital, University of Tampere, Finland
}

\section{A R T I C L E I N F O}

\section{Article history:}

Received 25 September 2017

Received in revised form 12 June 2018

Accepted 31 July 2018

\section{Key words:}

Necrotizing enterocolitis

Spontaneous intestinal perforation

Mortality

Surgical methods

Parenteral nutrition

\begin{abstract}
A B S T R A C T
Background: Necrotizing enterocolitis (NEC) and spontaneous intestinal perforation (SIP) are the most common abdominal surgical conditions in preemies. Associated mortality remains high and long periods of parenteral nutrition (PN) may be required. We assessed the developments in the outcomes of surgically treated NEC and SIP in the two largest Finnish neonatal intensive care units (NICU).

Methods: Retrospective observational study based on hospital records during 1986-2014. Main outcome measures were three-month survival during 1986-2000 compared with 2001-2014 and predictors of mortality. Results: Included were 225 patients (NICU A 131 and NICU B 94) with NEC in 142 (63\%) and SIP 83 (37\%). The median birth weight (BW) ( 870 vs $900 \mathrm{~g}$ ) and gestation age (GA) (27 vs 27 weeks, $p=0.96$ ) were similar in NEC and SIP. Small intestine was affected in $85 \%$ of NEC and 76\% of SIP patients $(p=0.12)$. In $5 \%$ of patients NEC was panintestinal. Median small intestinal loss was $25 \%$ in NEC and $4.0 \%$ in SIP ( $p<0.001)$. Ileocecal valve was resected in $29 \%$ of NEC and $14 \%$ of SIP patients $(p=0.01)$. Enterostomy was performed in $78 \%$ of patients and primary anastomosis in 18\%; $4 \%$ died of extensive NEC without definitive surgery. Overall survival was $74 \%$ (NEC 73\%, SIP 77\%, p = 0.48; NICU A 82\%, NICU B 65\%, p = 0.003). From 1986-2000 to 2001-2014 overall survival increased from 69 to $81 \%(\mathrm{p}=0.04)$. Treating NICU was the strongest predictor of survival, $\mathrm{RR}=2.8$ $(95 \% \mathrm{CI}=1.4-5.1), \mathrm{p}=0.003$.
\end{abstract}

Conclusions: Overall survival improved significantly from the early (1986-2000) to the late (2001-2014) study period. Strongest predictor of mortality was the treating neonatal intensive care unit.

Level of evidence: III.

() 2018 Elsevier Inc. All rights reserved.
Necrotizing enterocolitis (NEC) and spontaneous intestinal perforation (SIP) are the most common surgical conditions of premature infants [1-4]. Overall, they affect up to $2 \%$ of premature babies admitted into neonatal intensive care units (NICU) [4]. In very low birth weight (VLBW) and extremely low birth weight (ELBW) infants the prevalence of NEC and SIP is even higher varying from $5 \%$ to $10 \%$ [5] and NEC necessitates surgery in more than $50 \%$ of patients [6]. In patients with SIP or NEC with Bell stage III [7] surgery is almost invariably required. Mortality in surgically treated NEC varies from 30\% to 50\% [8-10]. Despite considerable recent advances in neonatal care, mortality of surgically treated NEC has not shown any significant changes [11]. Mortality after SIP, although also significant, is generally lower than in NEC $[12,13]$. The most significant gastrointestinal comorbidities in surgically

* Corresponding author at: Lautamiehentie 11 A 11, 01510 Vantaa, Finland. Tel.: + 358 400178372.

E-mail addresses: kristiina.karila@hus.fi (K. Karila),Annaleena.Anttila@pshp.fi (A. Anttila), iber.tarja@pshp.fi (T. Iber), mikko.pakarinen@hus.fi (M. Pakarinen), antti.koivusalo@hus.fi (A. Koivusalo). treated NEC and SIP include intestinal failure (IF) with associated cholestasis (IFAC) which affects as many as $42 \%$ of patients [14].

In this retrospective observational study we assessed the outcomes after surgically treated NEC and SIP in the two major level III NICUs in Finland with a total of 14,000 admissions during a 29-year study period from 1986 to 2014. Main outcome measure was three-month survival and the main focus of the study was to compare the outcomes during the early (1986-200) and the latter (2001-2014) study period. In addition we assessed clinical factors that associated with mortality. The results were expected to be useful both in the management of premature infants and in parent counseling.

\section{Methods}

The Ethical Review Boards of the both hospitals approved this study. The study was focused at the surgical management of NEC and SIP in prematures. Data were retrospectively collected from the hospital records, surgical reports and operation theater logbooks. Included were neonates who underwent surgery owing to acute deterioration caused by NEC (radiological signs consistent with of Bell stage III) [7] or SIP. 
Retrospective identification of nonsurgical NEC patients was not possible because of incomplete recording and uncertain differential diagnostics between septicemia and Bell stage I and II of NEC. Differential diagnosis between NEC and SIP was based on 1) preoperative diagnostics: SIP was always characterized by intestinal perforation indicated by free air in preoperative plain X-ray, NEC occurred with or without preoperatively diagnosed perforation with clinical signs of Bell stage II or III; 2) findings during the surgery: in SIP there was a perforation without or with a limited ( $\max .2 \mathrm{~cm}$ ) length of ischemic intestine while the rest of the intestine was unaffected, whereas in NEC there was a significant segment of ischemic intestine with or without perforation and sometimes several affected sites could be observed; 3 ) supportive data from pathologist's report of the resected intestine. Patients with intestinal conditions inconsistent with NEC of SIP were excluded, as well as patients who developed late stricture after conservative management of NEC. We excluded NEC related intestinal necrosis in term or almost term babies with cardiac disease or gastroschisis, which we think represents a different entity from NEC and SIP in premature infants.

In both centers NICU and pediatric surgical services were located in the same hospital building enabling prompt consultations. Surgeries were performed in NICU. Indications for surgery throughout the study period were perforation, or deteriorating general condition with clinical or radiological signs of intestinal disease. Excluding patients with poor general condition beyond resuscitation there were no absolute contraindications for surgery.

Survival was counted from the date of the primary operation. Survival during early (1986-2000) and late (2001-2014) study periods was compared. Predictive factors of survival included birth weight (BW), gestation age (GA), respiratory distress syndrome (RDS), diagnosis (NEC or SIP), relative loss of gestation age-adjusted percentage bowel length [15], type of operation (enterostomy vs primary anastomosis), reoperations, IF-associated cholestasis (IFAC) defined by conjugated bilirubin $>34 \mu \mathrm{mol} / \mathrm{l}(2.0 \mathrm{mg} / \mathrm{dl})$ for $\geq 2$ postoperative weeks with the patient receiving PN, treating NICU (NICU A, NICU B), Apgar scores, management of patent ductus arteriosus (PDA), intracerebral hemorrhage (IVH), significant heart defect other than PDA, duration of PN, septicemia, reoperations and duration of mechanical ventilation.

Statistical calculations were made with StatView 512 computer program (Brain Power, Calabasas, CA). Data are presented as medians with interquartile range (IQR) or frequencies. Cumulative survival and PNdependency were analyzed with Kaplan-Meier curves. Predictors of death and weaning off PN were analyzed with Cox proportional hazards regression analysis generating hazard ratios with $95 \%$ confidence interval. Statistically significant independent predictors were included in the multivariate model. P-values exceeding .05 were considered significant.

\section{Results}

\subsection{Clinical data}

In total 225 patients, 131 (58\%) from NICU A and 94 (42\%) from NICU B, were included. One hundred and forty-two (63\%) patients were operated for NEC and $83(37 \%)$ for SIP. Thus, approximately $1.6 \%$ of 14,000 patients admitted into NICUs underwent surgery for NEC $(1.0 \%)$ or SIP $(0.6 \%)$. Median birth weight was 880 (690-1100) g and gestation age 26 weeks 4 days (25weeks-28 weeks 2 days) Clinical data are outlined in Table 1.

NEC and SIP patients differed in their median age at first surgery 10 $(10-20)$ vs. 7 (5-9) days $(p=0.04)$ and in the percentage of patients who required medical or surgical treatment for PDA (45 vs 58\%, p = 0.04 ); differences in gender (females $29 \%$ vs $34 \%$ ), birth weight ( 870 vs $900 \mathrm{~g}$ ), gestation age (27 vs 27 weeks), Apgar scores, duration of mechanical ventilation ( 3.0 vs 2.6 weeks), incidence of cerebral hemorrhage (39\% vs $39 \%$ ) and incidence of moderate or severe RDS (48\% vs $48 \%$ ) were not statistically significant.

Location of the disease was small bowel in 120 (85\%) of NEC patients and in $63(76 \%)$ of SIP patients $(\mathrm{p}=0.78)$, whereas colon was involved in 20 (24\%) patients with SIP and in $11(16 \%)$ with NEC ( $p=0.01)$;
Table 1

Clinical data of 225 patients with surgically treated NEC or SIP.a

\begin{tabular}{|c|c|c|c|c|}
\hline & All & 1986-2000 & 2001-2014 & $\mathrm{P}$ \\
\hline Patients & 225 & 116 & 109 & \\
\hline NICU A & 131 & 66 & 65 & 0.68 \\
\hline NICU B & 94 & 50 & 44 & \\
\hline Birth weight, $g$ & $880(652-1015)$ & 883 (702-1090) & $870(652-1105)$ & 0.81 \\
\hline $\operatorname{ELBW}(\leq 1000 \mathrm{~g})$ & $143(64 \%)$ & $75(65 \%)$ & $68(62 \%)$ & 0.78 \\
\hline Gestation age, weeks & 26w 4d (I25-28) & $26 w 3 d(25-28)$ & 26 w 4d (25-28) & 0.68 \\
\hline Gender, female & $74(33 \%)$ & $41(29 \%)$ & $33(40 \%)$ & 0.48 \\
\hline \multicolumn{5}{|l|}{ Apgar score } \\
\hline $1 \mathrm{~min}$ & 5 (IQR 3-6) & 5 (IQR 2-6) & 6 (IQR 3-7) & 0.01 \\
\hline $10 \mathrm{~min}$ & 7(IQR 5-8) & 6(IQR 5-8) & 7 (IQR 6-8) & 0.06 \\
\hline Respirator weeks & $3.0(1.2-5.6)$ & $3.0(1.8-6.0)$ & $2.0(1.0-4.5)$ & 0.01 \\
\hline Severe / moderate RDS & $111(49 \%)$ & $69(59 \%)$ & $42(39 \%)$ & 0.002 \\
\hline \multicolumn{5}{|l|}{ Cerebral hemorrhage } \\
\hline none & 137 (49\%) & $69(59 \%)$ & $68(62 \%)$ & 0.68 \\
\hline Grade I-II & $41(18 \%)$ & $18(16 \%)$ & $23(21 \%)$ & 0.30 \\
\hline Grade III-IV & $37(16 \%)$ & $29(25 \%)$ & $18(17 \%)$ & 0.14 \\
\hline $\begin{array}{l}\text { PDA closed at birth or } \\
\text { afterwards } \\
\text { spontaneously }\end{array}$ & $115(51 \%)$ & $47(41 \%)$ & $68(62 \%)$ & 0.001 \\
\hline PDA, medical therapy & $68(30 \%)$ & $48(41 \%)$ & $20(18 \%)$ & 0.003 \\
\hline PDA, surgical therapy & $42(19 \%)$ & $21(18 \%)$ & $21(20 \%)$ & 0.86 \\
\hline Significant heart disease & $23(10 \%)$ & $6(5 \%)$ & $17(15 \%)$ & 0.01 \\
\hline NEC & 142 & $68(59 \%)$ & $74(68 \%)$ & $0.16^{\mathrm{a}}$ \\
\hline SIP & 83 & $48(41 \%)$ & 35 (32\%) & \\
\hline
\end{tabular}

$\mathrm{NICU}=$ neonatal intensive care unit, $\mathrm{PDA}=$ patent ductus arteriosus, $\mathrm{RDS}=$ respiratory distress syndrome.

a Refers to relative amount of NEC and SIP in 1986-2000 and 2001-2014.

moreover NEC was panintestinal in five (4\%) patients. Median length of small intestinal resection (\% of gestational age adjusted intestinal length) was 25 (IQR $12-44$ ) \% in NEC and 4 (IQR 2-7) \% in SIP $(\mathrm{p}<0.0001)$. Ileocecal valve was resected in $41(29 \%)$ patients with NEC and in $12(14 \%)$ patients with SIP ( $\mathrm{p}=0.01)$.

Comparison of clinical data from the periods 1986-2000 (116 patients) and 2001-2014 (109 patients) is presented in Table 1. Compared with patients from the period 1986-2000 patients from the period 2001-2014 had statistically significantly higher one-minute Apgar score, shorter median duration of mechanical ventilation, lower incidence of RDS, lower incidence of medical therapy for PDA, higher incidence of spontaneously closed PDA and higher incidence of heart diseases other than PDA. Admission into NICU A and NICU B, median birth weight, gestation age, gender, ten-minute Apgar score, incidence of cerebral hemorrhage, incidence of surgically treated PDA and incidence of NEC and SIP did not differ between the period 1986-2000 and 2001-2014.

Patients admitted into NICU A and NICU B differed in median birth weight ( 827 vs $900 \mathrm{~g}, \mathrm{p}=0.05$ ), gestation age (26 vs 27 weeks, $\mathrm{p}=$ 0.01 ) and in the incidence of PDA requiring surgical or medical treatment ( $60 \%$ vs $34 \%, \mathrm{p}=0.004)$.

\subsection{Operative characteristics}

The surgical procedure in the first operation was intestinal resection and enterostomy in 156 (69\%) (NEC $n=103,73 \%, \operatorname{SIP} n=53,64 \%, p=0.18$ ) and resection with primary anastomosis in $56(25 \%)$ patients (NEC $\mathrm{n}=28$, $20 \%$, SIP $n=28,34 \%, p=0.03$ ). Of the remaining 13 (6\%) patients five with NEC-induced panintestinal necrosis were deemed inoperable; two with spontaneously contained SIP initially underwent exploration only. In addition, six patients with NEC were deemed unfit for abdominal surgery and they underwent peritoneal drainage as the first surgery.

Comparison of operative characteristic from the periods 1986-2000 (116 patients) and 2001-2014 (109 patients) is presented in Table 2. The most marked change from the period $1986-2000$ to $2001-2014$ is the decreased use of primary anastomosis from $35 \%$ to $13 \%$ of patients $(\mathrm{p}=0.0002)$. The relative frequency of NEC and SIP, and location and length of affected intestine have remained unchanged. 
Table 2

NEC and SIP in 225 patients, comparison of two periods (1986-2000 and 2001-2014): primary location of disease, techniques in first surgery, median (IQR = interquartile range) extent of intestinal resection (\% of gestation age adjusted length).

\begin{tabular}{lllll}
\hline & All & $1986-2000$ & $2001-2014$ & $\mathrm{p}$ \\
\hline Patients & 225 & 116 & 109 & \\
NEC & 142 & $68(59 \%)$ & $74(68 \%)$ & 0.16 \\
SIP & 83 & $48(41 \%)$ & $35(32 \%)$ & \\
Location & $184(82 \%)$ & $97(84 \%)$ & $87(80 \%)$ & 0.35 \\
Small intestine & $36(16 \%)$ & $16(14 \%)$ & $20(18 \%)$ & 0.19 \\
Colon & $5(2 \%)$ & $3(3 \%)$ & $2(2 \%)$ & 0.67 \\
Panintestinal (NEC) & & & & \\
Technique in first surgery, all & $156(69 \%)$ & $70(60 \%)$ & $86(79 \%)$ & $\mathbf{0 . 0 0 4}$ \\
-resection and enterostomy & $156(25 \%)$ & $41(35 \%)$ & $15(13 \%)$ & $\mathbf{0 . 0 0 0 2}$ \\
-resection and primary repair & $56(5 \%)$ & $5(5 \%)$ & $8(8 \%)$ & 0.43 \\
-drainage or exploration & $13(6 \%)$ & & & \\
NEC & & $42(62 \%)$ & $61(82 \%)$ & $\mathbf{0 . 0 1}$ \\
-resection and enterostomy & $103(73 \%)$ & $21(31 \%)$ & $7(10 \%)$ & $\mathbf{0 . 0 0 1}$ \\
-resection and primary repair & $28(20 \%)$ & $5(7 \%)$ & $6(8 \%)$ & 0.68 \\
-drainage or exploration & $11(7 \%)$ & $5(28)$ & \\
SIP & & & & \\
-resection and enterostomy & $53(64 \%)$ & $28(58 \%)$ & $25(71 \%)$ & 0.25 \\
-resection and primary repair & $28(34 \%)$ & $20(42 \%)$ & $8(33 \%)$ & 0.10 \\
-drainage or exploration & $2(2 \%)$ & 0 & $2(6 \%)$ & 0.17 \\
Resection of small intestine \% & & & & \\
- NEC & $21(10-40)$ & $21(10-36)$ & $22(10-50)$ & 0.76 \\
- SIP & $3.0(0.0-5.0)$ & $2.9(0.0-4.5)$ & $3.0(0.3-6.7)$ & 0.77 \\
Resection of colon \% & & & & \\
- NEC & $0.0(0.0-12)$ & $0.0(0.0-12)$ & $0.0(0.0-13)$ & 0.79 \\
- SIP & $0.0(0.0-2.5)$ & $0.0(0.0-2.5)$ & $0.0(0.0-4.5)$ & 0.24 \\
Ileocecal valve resected & & & & \\
-NEC & $41(29 \%)$ & $20(29 \%)$ & $21(28 \%)$ & 0.99 \\
-SIP & $12(14 \%)$ & $7(15 \%)$ & $5(14 \%)$ & 0.99 \\
\hline p & & & & \\
\hline
\end{tabular}

$\mathrm{p}$ in the right column refers to the difference between 1986-2000 and 2001-14.

$* \mathrm{p}=0.04$, refers to the overall difference in resection of ileocecal valve in NEC and SIP.

Excluding the final enterostomy closure, reoperations were performed to 87 (39\%) of patients including 64 (45\%) of 142 NEC patients and $23(27 \%)$ of 83 SIP patients $(\mathrm{p}=0.01)$. Overall enterostomy (156 patients) and primary anastomosis (56 patients) as the first surgery required a reoperation in 61 (39\%) and 22 (39\%) patients, respectively $(\mathrm{p}=0.87)$. Enterostomy (101 patients) and primary anastomosis (27 patients) in NEC required a reoperation in $45(43 \%)$ and $14(52 \%)$ patients, respectively $(\mathrm{p}=0.52$ ). Enterostomy (55 patients) and primary anastomosis (26 patients) in SIP required a reoperation in 15 (27\%) and $7(27 \%)$ patients $(\mathrm{p}=0.99)$. Indications for reoperations were recurred or progressive disease $(n=24)$, obstruction $(n=27)$, anastomotic dehiscence ( $n=15)$, problems with enterostomy $(n=7)$, and suspected complication $(\mathrm{n}=12)$. The percentage of reoperated patients did not change from 1986-2000 (41\%) to 2001-2014 (36\%) ( $p=0.41$ ).

Eventually, 176 (78\%) patients ended up with enterostomy, 40 (18\%) remained with primary anastomosis and 8 (4\%) died without any attempt on curative resection. Of the 176 patients with enterostomy, 132 survived to undergo final enterostomy closure, which occurred after a median 9.0 of (IQR 5.1-16) weeks.

Surgical techniques in NICU A and NICU B were similar (enterostomy $79 \%$ and $70 \%$, primary anastomosis $21 \%$ and $30 \%, p=0.19$ ).

\section{Survival and predictors of mortality}

\subsection{Survival}

\subsubsection{Overall survival and survival in NEC and SIP}

Overall three-month survival (1986-2014) including both NEC and SIP in NICU A and B together was $74(95 \% \mathrm{CI}=69-80) \%$. In NEC patients survival was $73(95 \% \mathrm{CI}=65-80) \%$ and in SIP $77(95 \% \mathrm{CI}=68-86) \%$, $(\mathrm{p}=0.10)$ (Fig. 1). Majority of the deaths occurred within two weeks after the first surgery; survival at two weeks was $79(95 \% \mathrm{CI}=74-84)$ $\%$. From three months to hospital discharge overall survival decreased only from $74(95 \% \mathrm{CI}=69-80) \%$ to $73(95 \% \mathrm{CI}=67-79) \%(\mathrm{p}=0.95)$.

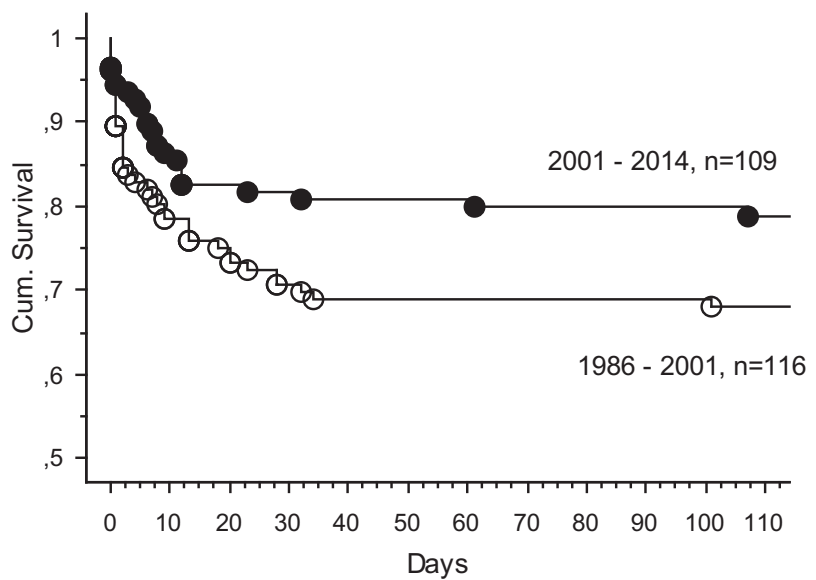

Fig. 1. Three-month overall mortality of patients with necrotizing enterocolitis or spontaneous intestinal perforation during 1986-2000 [68 (95\% CI $=60-77) \%]$ and 2001-2014 [80 (95\% CI = 71-87) \%], Log-Rank Mantel-Cox, $\mathrm{p}=0.02$.

Overall three-month survival of ELBW (BW $<1000 \mathrm{~g}$ ) patients ( $\mathrm{n}=$ 143) was $68 \%$ ( $95 \% \mathrm{CI}=59-75 \%$ ). Causes of death (available from NICU A, 31 patients) were direct consequence of NEC or SIP $(n=15) 45 \%$, prolonged cholestasis $(n=6) 19 \%$, respiratory collapse $(n=3) 10 \%$, intracerebral hemorrhage $(n=2), 7 \%$, multiorgan failure $(n=2) 7 \%$, extreme prematurity $(n=2) 7 \%$ and septicemia $(n=1) 3 \%$.

\subsubsection{Overall survival by periods $1986-2000$ vs 2001-14}

From the period 1986-2000 (116 patients) to the period 2001-2014 (108 patients) the overall survival improved from 68\% (95\% CI = $61-75) \%$ to 80 (95\% CI $=72-87) \%(\mathrm{p}=0.02)$ (Fig. 1$)$. The overall survival in NEC from 1986 to $2000,69 \%(95 \% \mathrm{CI}=58-80)$, to $2001-14,77 \%$ (95\% CI $=65-86),(p=0.30)$ did not improve statistically significantly whereas in SIP there was statistically significant improvement from 69 $(95 \% \mathrm{CI}=56-82) \%$ to $89(95 \% \mathrm{CI}=78-99) \%(\mathrm{p}=0.04)$. Survival of ELBW patients from $64 \%(95 \% \mathrm{CI}=53-75)$ to $71 \%(95 \% \mathrm{CI}=60-81)$ did not improve statistically significantly $(\mathrm{p}=0.28)$.

Compared to patients from the period 2001-2014, patients from $1986-2000$ had a lower prevalence of heart defects $(5.0 \%$ vs $16 \%, \mathrm{p}=$ 0.03 ) and more frequent use of primary anastomosis (34\% vs $12 \%$, $\mathrm{p}=0.001$ ), whereas birth weight, gestation age, Apgar scores, percentage of resected small bowel, duration of PN, incidences of RDS, intraventricular cerebral hemorrhage (IVH) and sepsis, treatment of PDA, distribution of patients between treating centers and relative number of patients with NEC and SIP were similar.

\subsubsection{Predictors of mortality}

Predictors of mortality are outlined in Table 3. In multivariate analysis only the treating NICU predicted survival.

Assessment of the period 1986-2000 and 2001-2014 separately showed that of the tested factors only septicemia $\mathrm{RR}=1.5(95 \% \mathrm{CI}=$ $1.0-2.4),(\mathrm{p}=0.04)$ predicted mortality during 1986-2000. During 2001-2014 mortality was predicted by BW $<750$ g, RR $=8.6(95 \%$ $\mathrm{CI}=2.7-28),(\mathrm{p}=0.0003), \mathrm{GA}<26$ weeks $6.2(95 \% \mathrm{CI}=2.1-19)$, $(\mathrm{p}=0.001), \mathrm{RDS}, \mathrm{RR}=7.4(95 \% \mathrm{CI}=1.5-35), \mathrm{p}=0.01$ and $\mathrm{NICU}$ $\mathrm{RR}=3.0(95 \% \mathrm{CI}=1.2-8.0), \mathrm{p}=0.03$ (univariate analysis) and $\mathrm{BW}<750 \mathrm{~g} R \mathrm{RR}=4.3(1.1-16),(\mathrm{p}=0.03)$. Although the choice of surgical techniques shifted to increased use of enterostomies during 2001-2014, use of enterostomy was not associated with increased survival, $\mathrm{RR}=1.9(95 \% \mathrm{CI}=0.6-5.8),(\mathrm{p}=0.23)$.

\subsubsection{Survival by center}

From 1986 to 2014 overall survival in NICU A and NICU B was 81 $(95 \% \mathrm{CI}=74-88) \%$ and $63(95 \% \mathrm{CI}=53-73) \%(\mathrm{p}=0.002)$. From 1986-2000 to 2001-2014 survival in NICU A rose from 74 (64-85) \% 
Table 3

Factors predicting mortality at three months in 225 patients with surgically treated NEC or IIP in logistic regression analysis.a

\begin{tabular}{|c|c|c|c|c|}
\hline \multirow[t]{2}{*}{ Factor } & \multicolumn{2}{|l|}{ Univariate analysis } & \multicolumn{2}{|l|}{ Multivariate analysis } \\
\hline & Risk ratio (RR) & $\mathrm{p}$ & Risk ratio (RR) & $\mathrm{p}$ \\
\hline Birth weight $<750 \mathrm{~g}$ & $2.3(95 \% \mathrm{CI}=1.2-4.2)$ & 0.01 & & \\
\hline Gestational age $<26$ weeks & $2.2(95 \% \mathrm{CI}=1.2-4.0)$ & 0.01 & & \\
\hline RDS & $3.3(95 \% \mathrm{CI}=1.3-8.6)$ & 0.01 & & \\
\hline NICU & $2.4(95 \% \mathrm{CI}=1.3-4.4)$ & 0.005 & $2.8(95 \% \mathrm{CI}=1.5-5.3)$ & 0.003 \\
\hline Gender & $0.9(95 \% \mathrm{CI}=0.5-1.8)$ & 0.81 & & \\
\hline Mothers age & $0.9(95 \% \mathrm{CI}=0.9-1.0)$ & 0.27 & & \\
\hline Apgar points $1^{\prime}$ and $10^{\prime}$ & $1.0(95 \% \mathrm{CI}=0.8-1.2)$ & $0.75-0.79$ & & \\
\hline Small bowel resection (\%) & $1.0(95 \% \mathrm{CI}=1.0-1.0)$ & 0.37 & & \\
\hline IVH Grade I-II & $0.8(95 \% \mathrm{CI}=0.4-1.9)$ & 0.64 & & \\
\hline IVH Grade III-IV & $1.1(95 \% \mathrm{CI}=0.5-2.3)$ & 0.78 & & \\
\hline PDA, Surgery & $0.9(95 \% \mathrm{CI}=0.4-2.1)$ & 0.88 & & \\
\hline PDA, Medical & $1.6(95 \% \mathrm{CI}=0.8-3.2)$ & 0.15 & & \\
\hline Septicemia $^{\mathrm{a}}$ & $1.6(95 \% \mathrm{CI}=1.1-2.4)$ & 0.01 & & \\
\hline NEC & $1.2(95 \% \mathrm{CI}=0.7-2.3)$ & 0.52 & & \\
\hline Primary repair & $0.6(95 \% \mathrm{CI}=0 .-1.3)$ & 0.20 & & \\
\hline Enterostomy & $0.8(95 \% \mathrm{CI}=0.4-1.6)$ & 0.56 & & \\
\hline Reoperations & $1.2(95 \% \mathrm{CI}=0.7-2.2)$ & 0.52 & & \\
\hline Duration of PN & $1.0(95 \% \mathrm{CI}=1.0-1.1)$ & 0.17 & & \\
\hline IFAC & $1.2(95 \% \mathrm{CI}=0.4-4.1)$ & 0.72 & & \\
\hline
\end{tabular}

a Septicemia that occurred after $1^{\text {st }}$ surgery

to $88 \%(80-96) \%$ and in NICU B from $58(95 \% \mathrm{CI}=44-72) \%$ to 66 ( $95 \%$ CI $52-80) \%(p=0.23)$. In NICU A survival in NEC rose from $70(95 \%$ $\mathrm{CI}=54-84) \%$ to $86(95 \% \mathrm{CI}=76-97) \%(\mathrm{p}=0.04)$, and in NICU B the survival in SIP rose from $45(95 \% \mathrm{CI}=23-67) \%$ to $87(95 \% \mathrm{CI}=$ $69-103) \%(p=0.02)$.

Hospital admission records showed that during 1986-2000 the overall mortality in NICUA was 9.6\% (595 / 6199 admitted patients), and during 2001-2014, 4.3\% (283 / 6635 patients). Overall mortality (NEC and SIP) during the respective periods, 31 and 19\%, exceeded general mortality 3.2 and 4.4 fold.

\subsection{Parenteral nutrition and IFAC}

Of 225 patients 157 (70\%) (NEC $n=98$ and SIP $n=59)$ had at least two weeks of postoperative PN. Of the 157 patients IFAC developed in 64 (41\%). The median duration of PN was 26 (IQR 14-55) days without significant difference between NEC 27 (IQR 14-60) days) and SIP 25 (IQR 13-45) days $(\mathrm{p}=0.32)$. One and three months after surgery 99 (63\%) and 34 (22\%) of survived patients were dependent on PN. PN dependency in NEC and SIP was 59\% and 52\% at one month $(p=0.43)$ and $26 \%$ and $11 \%(\mathrm{p}=0.01)$ at three months. Incidence of IFAC in NEC was (47/98) $48 \%$ and in SIP 18/59 (31\%) ( $p=0.04)$. Duration of IFAC from start to resolution was 92 (61-121) days in NEC and 47 (38-121) days in SIP $(p=0.30)$. Dependency on PN or development of IFAC did not associate with overall mortality, but all eight patients $(\operatorname{NEC} n=6$, SIP $n=2$ ) with unresolved IFAC died.

\section{Discussion}

In the present study we analyzed the outcomes of surgically treated NEC and SIP over a 29-year period, from 1986 to 2014 in the two largest Finnish NICUs. We found that during the study period, of admitted neonates approximately $1.0 \%$ underwent surgery for NEC and $0.6 \%$ for SIP. During the 29-year study period the management of prematures has undergone changes and advances have been made, but indications for surgery for NEC and SIP have remained unchanged. In the present study the gestational age and birth weight of the surgically managed patients were similar in the early and late periods of the study period and still remained comparable with the respective figures in recent series of NEC and SIP. We focused on the development of the survival from the early study period (1986-2000) to the latter period (2001-2014) and our main finding is an improvement of the overall three-month survival during the latter period of the study (2001-14). Overall mortality was highest during the two weeks following the first surgery and from three to six months after surgery mortality increased very little. Main factors associated with mortality during the 29-year study period were low birth weight, low gestation age, respiratory distress syndrome and the attending NICU. When the early and late study periods were studied separately we found that these predictive factors were significant during the latter study period whereas none were significant during the early period. Although the general mortality in NICUA more than halved from the early to the late study period, mortality from NEC and SIP remained 3-4 fold compared with general mortality. No improvement occurred in the survival of patients with ELBW. Main shortcomings of the study were the retrospective design and relatively low number of patients in relation to the long observation period. Because of defective recording patients who had medical treatment of NEC could not be identified and had to be excluded from the study. In addition we were not able to assess the effects of preoperative clinical parameters and hospital transfers on survival.

In our series the overall survival of NEC (73\%) and SIP (77\%) and the survival of ELBW neonates (68\%) were comparable with contemporary studies by Hull et al. [2], Fitzgibbons et al. [16], Fullerton et al. [17] and Stey et al. [18]. Not unexpectedly low birth weight $(<750 \mathrm{~g})$ and gestation age $(<26 \mathrm{w})$ were independent predictors of mortality and these findings concur with previous findings by Hull et al. [2], Fitzgibbons et al. [16], Guner et al. [19], Kessler et al. [20] and Allin et al. (2017). We found that moderate or severe RDS was common in patients with NEC and SIP and independently predicted mortality. In concordance with our results Okyuama et al. [13] reported RDS as a strong risk factor for SIP and Blakely et al. [21] found that in patients with NEC and SIP requirement of high frequency oscillating ventilation (HFOV) or a high positive inspiratory pressure predicted death.

We found that the strongest factor that predicted the overall survival was the attending NICU. Survival in the two study centers differed significantly. Our data do not directly explain the reason for different results between the two centers. It may be speculated that of the two centers with equal facilities for neonatal care NICU A is larger and has a larger case load of both premature patients and patients with NEC and SIP and this probably contributes to the better results. As a national tertiary cente the pediatric surgical service of NICU has wide knowledge in the management of intestinal failure which benefits also the management of NEC and SIP. There may also be differences in the general condition of the patients referred with a manifest NEC or SIP. The outcome of NEC and SIP depends on multiple factors related to patient management and transfer [16]. NICU A is responsible for a relatively compact 
and more densely populated area than NICU B and neonates referred to NICUB may be at disadvantage because of larger transfer distances. Because of the emergent nature of the two conditions centralization of the surgery of NEC and SIP does not seem possible.

In our series definitive surgical procedure was eventually attempted in nearly $95 \%$ of patients, which is a high figure compared with previous series reporting almost 30\%-50\% rate of peritoneal drainage as the primary or only procedure, $[2,21]$. Our good results benefitted from effective management by the neonatologists rendering the majority of our patients fit for definitive surgery instead of mere peritoneal drainage.

In NEC and SIP survival figures exceeding $60 \%$ have been reported since early 1980 s $[4,22,23]$. In EWBL infants, however, $50 \%$ survival of NEC was reported as recently as 2005 by Blakely et al. [21]. More recent NEC survival figures vary from $65 \%$ to $69 \%[2,18]$. In a recent large series of almost 9000 patients, mortality of surgical NEC was found to plateau at $30 \%$ in patients whose birth weight exceeded $750 \mathrm{~g}$. In the present study the reasons for gradual improvement in the outcome of NEC and SIP are not obvious. Clinical characteristics of the patients were unchanged during the 29-year study period.

The ideal operative techniques for NEC and SIP are disputed. There are no studies with adequate power and proper construction to show the benefits of primary anastomosis over enterostomy or vice versa $[24,25]$. We favor enterostomy in both NEC and SIP because the risks of primary anastomosis - dehiscence and obstruction - are avoided in a critically ill patient. In addition, enteral feeding can be started soon after surgery and the distal bowel utilized by refeeding the stools through the efferent stomy. In a premature a primary anastomosis may malfunction even after proper healing and intestinal function may be further hindered by poor motility of the colon with a net result of an unnecessary delay in enteral feeding and prolonged exposure to PN and the associated complications. De Haro Jorge et al. [26] reported that patients with SIP enterostomy were safer than primary anastomosis from the point of life-threatening complications. Although the finding of de Haro Jorge et al. [26] cannot directly be supported by our data our present clinical experience supports their view. In the present study primary anastomosis was used more often during 1986-2000 than during 2001-14, but despite frequent failures and conversions to enterostomy we cannot deduce that added mortality could be associated with primary anastomosis. For proper comparison of enterostomy and primary anastomosis the construction of the present study was not adequate.

Advances in neonatal care may be a factor that has improved the overall survival of our patient with NEC and SIP towards the latter period. However, we found that the risk of mortality at the latter period was associated more with extreme prematurity - low gestational age, low birth weight and RDS than in the earlier period. In patients admitted in NICU death from NEC and SIP remained 3-4 fold compared with the mortality of all causes. In majority of cases death occurred within 2 weeks after the surgery and was directly associated with intestinal disease. After surgery for NEC and SIP development of IF was not uncommon. After the first postoperative month we found that more than $50 \%$ and after 3 months more than 20\% of the patients were dependent on PN. In a study by Elfvin et al. (2015), PN dependency at 3 months in surgical NEC patients was slightly lower at $15 \%$. Even in medically treated NEC patients PN-dependency at one month has been reported at $25 \%$ [6]. IF, long PN, IFAC and sepsis are associated with poor prognosis in infants who survive SIP and NEC. In the present series majority of the mortality occurred soon after surgery and the effect of factors associated with IF became not significant.
In a retrospective study of 225 Finnish patients with surgical NEC and SIP overall survival was 74\%. Survival in NEC and SIP did not differ statistically significantly. From 1986-2001 to 2001-2014 marked improvement of overall survival was observed. Death was predicted by birth weight under $750 \mathrm{~g}$, gestational age $<26$ weeks, respiratory distress syndrome and attending NICU. The reasons of the improved survival were not evident but we suggest the roles of improved premature care and improved insight into the management of the intestinal disease.

\section{References}

[1] Hunter C, Chokshi N, Ford H. Evidence vs experience in the surgical management of necrotizing enterocolitis and focal intestinal perforation. J Perinatol 2008:28:S14-7.

[2] Hull M, Fisher J, Gutierrez I, et al. Mortality and management of surgical necrotizing enterocolitis in very low birth weight neonates: a prospective cohort study. J Am Coll Surg 2014;6:218.

[3] Boston V. Necrotising enterocolitis and localized intestinal perforation: different diseases or ends of a spectrum of pathology. Pediatr Surg Int 2006;22:477-84.

[4] Gregory K, DeForge C, Natale K, et al. Necrotizing enterocolitis in the premature infant: neonatal nursing assessment, disease pathogenesis, and clinical presentation. Adv Neonatal Care 2011;11:155-66.

[5] Patel B, Shah J. Necrotizing enterocolitis in very low birth weight infants: a systematic review. ISRN Gastroenterol 2012;2012:562594.

[6] Elfvin A, Dinsdale E, Wales P, et al. Low birthweight, gestational age, need for surgical intervention and gram-negative bacteraemia predict intestinal failure following necrotizing enterocolitis. Acta Paediatr 2015;104:771-6.

[7] Coran A, Adzick S, Krummel T, et al. Pediatric surgeryseventh edition; 2012 chapter 94, pg 1187. Necrotizing Enterocolitis.

[8] Rees C, Eaton S, Pierro A. Trends in infant mortality from necrotizing enterocolitis in England and Wales and the USA. Arch Dis Child Fetal Neonatal Ed 2008;93:395-6.

[9] Palmer S, Biffin A, Gamsu H. Outcome of neonatal necrotizing enterocolitis: results of the BAPM/CDSC surveillance study, 1981-84. Arch Dis Child 1989;64:388-94.

[10] Camberos A, Patel K, Applebaum H. Laparotomy in very small premature infants with necrotizing enterocolitis or focal intestinal perforation: postoperative outcome. J Pediatr Surg 2002;12:1692-5.

[11] Thakkar H, Lakhoo K. The surgical management of necrotizing enterocolitis (NEC) Early Hum Dev 2016;97:25-8.

[12] Rovin J, Rodgers B, Burns C, et al. The role of peritoneal drainage for intestinal perforation in infants with and without necrotizing enterocolitis. J Pediatr Surg 1999;34: 143-7.

[13] Okuyama $\mathrm{H}$, Kubota A, Oue $\mathrm{T}$, et al. A comparison of the clinical presentation and outcome of focal intestinal perforation and necrotizing enterocolitis in very-lowbirth-weight neonates. Pediatr Surg Int 2002;18:704-6.

[14] Duro D, Kalish L, Johnston P, et al. Risk factors for intestinal failure in infants with necrotizing enterocolitis: a Glaser pediatric research network study. J Pediatr 2010;157:203-8.

[15] Struijs M-C, Diamond I, de Silva N, et al. Establishing norms for intestinal length in children. J Pediatr Surg 2009;44:933-8.

[16] Fitzgibbons S, Ching Y, Carpenter J, et al. Mortality of necrotizing enterocolitis expressed by birth weight categories. J Pediatr Surg 2009;44:1072-6.

[17] Fullerton B, Sparks E, Morrow K, et al. Hospital transfers and patterns of mortality in very low birth weight neonates with surgical necrotizing enterocolitis. J Pediatr Surg 2016;51:932-5

[18] Stey A, Barnert E, Tseng C-H, et al. Outcomes and costs of surgical treatments of necrotizing enterocolitis. Pediatrics 2015;5:135.

[19] Guner Y, Friedlich P, Epi M, et al. State-based analysis of necrotizing enterocolitis outcomes. 2009;157:21-9.

[20] Kessler U, Schulte F, Cholewa D, et al. Outcome in neonates with necrotizing enterocolitis and patent ductus arteriosus. World J Pediatr 2016;12(1):55-9.

[21] Blakely M, Tyson J, Lally K, et al. Laparotomy versus peritoneal drainage for necrotizing enterocolitis or isolated intestinal perforation in extremely low birth weight infants: outcomes through 18 months adjusted age. Pediatrics 2006;4:117.

[22] Pokorny W, Garcia-Prats J, Barry Y. J Pediatr Surg 1986;21(12):1149-54.

[23] Kliegman R, Fanaroff A. Neonatal necrotizing enterocolitis: a nine-year experience. II. Outcome assessment. Am J Dis Child 1981;135(7):608-11.

[24] Downard C, Renaud E, St. Peter S, et al. Treatment of necrotizing enterocolitis: an American pediatric surgical association outcomes and clinical trials committee systematic review. J Pediatr Surg 2012;47:2111-22.

[25] Haricharan R, Gallimore J, Nasr A. Primary anastomosis or ostomy in necrotizing enterocolitis? Pediatr Surg Int 2017;33:1139-45.

[26] De Haro Jorge I, Ortells J, Cazalla A, et al. Long term outcome of preterm infants with isolated intestinal perforation: a comparison between primary anastomosis and ileostomy. J Pediatr Surg 2016;51:1251-4. 